\title{
Wireless Noise Characteristics on Gearboxes Due to Speed Variations
}

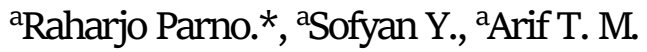 \\ aMechanical Engineering Department, Politeknik Negeri Bandung, Bandung 40559, Indonesia \\ Received 12 April 2019; accepted 12 December 2019
}

\begin{abstract}
Noise inspection is a predictive maintenance technique that is used to determine machine condition. The noise inspection can be done offline and online. Online noise inspection, which is far away from the object, is performed in the control center room. This monitoring system requires a complicated installation and long cables. The complexity of installation can be overcome by implementing a wireless noise inspection system. Wireless noise monitoring system for machinery condition monitoring still lacks information. Therefore, it is necessary to develop a wireless noise monitoring system. The result of wireless noise testing data on the machine is justified through the analysis of noise testing data of wired system. The research objective was to create a wireless noise measurement that is applied on a gearbox that is equipped with a data acquisition system that operates at a constant load and 5 variations of speed. Comparative analysis is used to justify the noise amplitude, time domain, and frequency domain of wireless and cabled measurements. The final test result indicates that the noise and wireless spectrum signals match the noise spectrum and signals using a cable. The highest amplitude lies at 12-13 of a fundamental frequency at a low frequency and at 30 of a fundamental frequency at a high frequency.
\end{abstract}

\section{KEYWORDS}

Noise

Gearboxes

Speed variation

Fundamental frequency

\section{INTRODUCTION}

Noise inspection is a predictive maintenance technique that aims to determine the condition of a machine or equipment through the noise condition that occurs on the machine. Noise monitoring has been widely applied, but this is mainly for safety and health purposes. Noise inspection for machine conditions is still very inadequate and it is not yet well-established like vibration monitoring. Huskey A and van Dam J (2010) investigated an acoustic noise on wind turbine generator systems for understanding the noise level or Sound Power Level as a function of wind speed (Huskey et. al., 2010). Metwally and his colleague (2011) investigated the noise characteristics of air conditioners in a vehicle for passenger comfort (Metwally et. al., 2011).

Noise monitoring has been carried out but this is to determine the noise level associated with health and safety. The noise monitoring for determining the condition of an engine or equipment has not yet been established well. The noise or airborne sound monitoring has not been widely applied in the industry, whereas the physical, signal and spectral characteristics the noise are

*Corresponding Author: parno_raharjo@polban.ac.id

IJATR is licensed under a Creative Commons Attribution-Share Alike 4.o International License. 
similar to those of vibration. Nevertheless, noise or acoustic monitoring has been conducted by some researchers. However, information is still limited, and further research is needed.

Ramroop et. al. (2001) implemented a monitoring of the condition of the gearbox system using airborne acoustics which resulted in the acoustic monitoring turning better than the vibration method. In addition, they created an excellent guide for implementing the monitoring of acoustic conditions (Ramroop et. al., 2001). Bayidar (2001) conducted a comparative study of vibration and acoustic measurements to detect a gear damage. The results showed that acoustics can be used to detect a gear damage effectively such as gear crack, localized wear, and broken teeth (Bayidar et. al., 2001). Cook (2012) performed acoustic monitoring for machine tool conditions through a noise jet. The results showed that there is a real relationship between cutting tool wear and noise signals on the machine tools (Cook, 2012).

Raharjo and his colleagues (2012) conducted a comparative study on the monitoring of the condition of a scratched self-aligning spherical journal bearing using a surface vibration, airborne sound, and acoustic emission (AE) analysis. The results showed that the signal and spectrum of the three measurements can differentiate between normal journal bearing and scratched journal bearing. Airborne sound showed that the highest amplitude occurs at a frequency of $550 \mathrm{OHz}$ and the highest amplitude of $\mathrm{AE}$ occurs at a frequency of $25 \mathrm{kHz}$. $\mathrm{AE}$ indicated the highest sensitivity (Raharjo et. al., 2012).

From the various studies above, it can be concluded that noise analysis can be used to detect the failure of the machine. However, the noise signal and spectrum noise characteristics of component failure cannot be identified well. Therefore, it is necessary to study noise characteristics to detect engine damage more widely and more intensively.

A noise on an engine can come from any vibrating engine component. The noise transmits as a wave through the air environment and reaches the sensor or the listener. The noise is a variation of local pressure moving through the medium of the material, the transmission speed depending on the nature of the material itself (Barron, 2003).

Noise from machining or rotary engines may be an aerodynamic, electromagnetic and structural noise. Each component generates noise at a specific frequency associated with the natural frequency (Kim et. al., 2010).

In industry, aerodynamic noise is generally generated by the pneumatic discharge system. However, aerodynamic noise also arises where there exists a relatively large velocity between an object with the surrounding air, for instant, in the ventilator blades. Electromagnetic noise is generated by electromagnetic forces that are generated by an interaction between magnetic and an electric power.

Noise that is caused by a vibration arises in solid, liquid and gas and depends on the transmission media. If a solid object vibrates, the structure of air borne sound is transmitted around its gas. The vibrating solid body then becomes the source of the noise that causes airborne sound (Schenk). Noise is also generated by friction. Friction on two different materials in the sliding action affects the noise and vibration energy (Rorrer, 2002).

The noise inspection can be offline and online. Online noise inspection, which is far away from the object, is performed in the control center room. This monitoring system requires a complicated installation and long cables. The complexity of installation can be overcome by 
implementing a wireless noise inspection system. Wireless noise monitoring system for machinery condition monitoring still lacks information. Therefore, it is necessary to develop a wireless noise monitoring system. However, wireless vibration monitoring has been investigated by only several researchers. Wang and his colleagues conducted vibration monitoring research using commercial wired and wireless monitoring systems on a bridge and the results showed that there is a strong match between cabled and wireless system measurement data (Wang et. al., 2006).

Lutfhi and his friends (2010) developed an integrated wireless acceleration sensor that can be mounted on the shaft. The experimental results showed that wireless accelerometer signals have less noise and create a small detection error (Lutfhi et. al., 2010). Because vibration and noise have the same characteristics, wireless or wired noise can be used to determine the condition of the machine.

\section{METHODOLOGY}

\section{Test Methods}

Noise testing is done on the gearbox speed variations of 300/60 rpm, 450/90 rpm, 600/120 rpm and 1000/200 rpm. Instruments that are used are created using wireless and standard wired microphones. These instruments are connected to a data acquisition channel. The test installation is shown in the following figure:

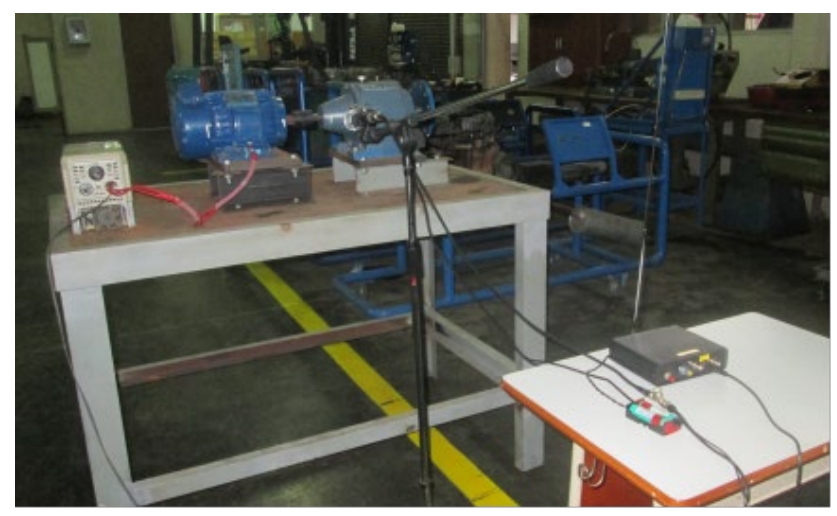

Figure 1. Microphone and transmitter are installed on a gearbox transmission

\section{Instrumentation and Data acquisition systems}

The tested instrumentation is a wireless communication device using an FSK modem that is equipped with a radio transceiver working on UHF with a power of about 1 Watt. This device consists of main components of the TCM3105 IC, which can work up to 1200 bps in a full-duplex mode. Transmitter and receiver modules are shown in the following figure: 

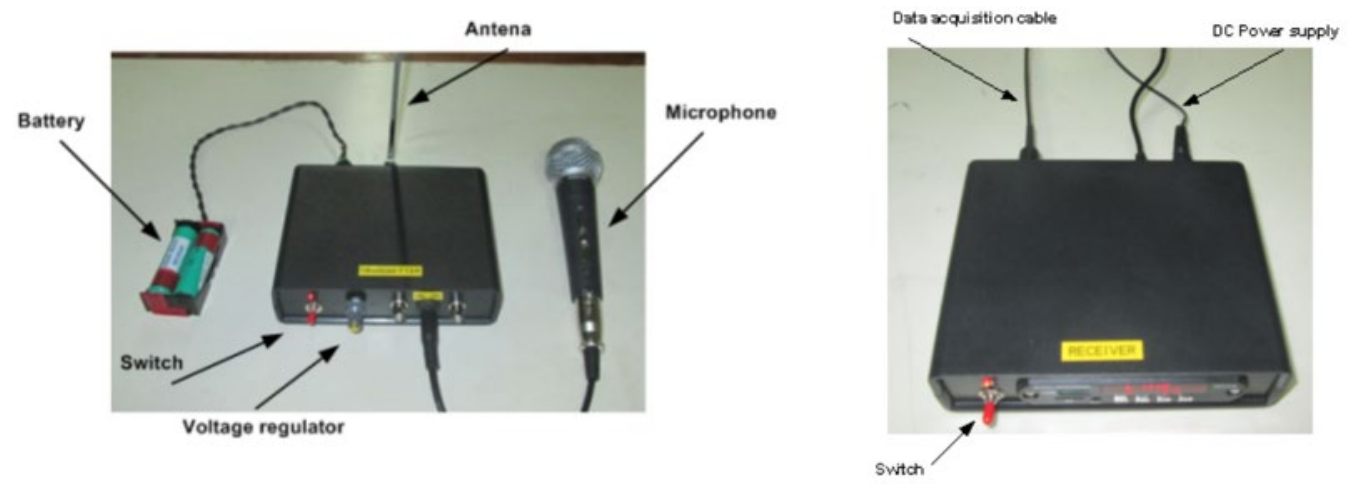

Figure 2. Created transmitter and receiver

Testing is done with a cable system as a validation device, and wireless system as a tested device. ICP microphone BAST YG 20107067 with sensitivity of $49.5 \mathrm{mV}$ / Pa and frequency range of $20 \mathrm{~Hz}-100 \mathrm{kHz}$ is used in this experiment (GST, 2010). Sinocera YE 6231 Dynamic Signal Analyzer with 4 channels with $96.0 \mathrm{kHz}$ sampling rate per channel is used for data acquisition. Instrumentation scheme of wired and wireless also data acquisition are shown in the following figure:

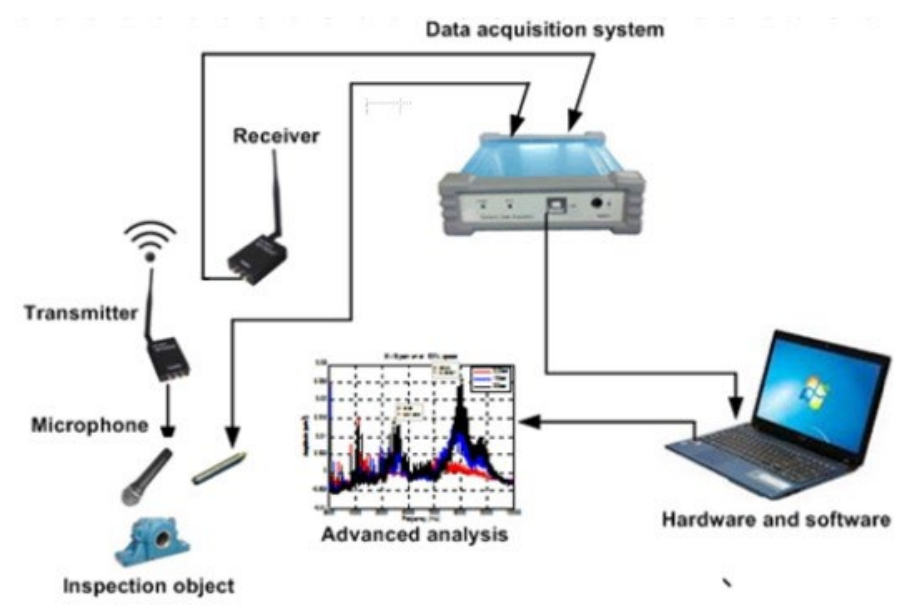

Figure 3. Scheme of the wired and wireless noise monitoring system

\section{RESULT AND DISCUSSION}

\section{Noise Signal Result}

The result of noise signals on a standard wired microphone and the created wireless at a 600/120 rpm speed is shown in the following figure: 


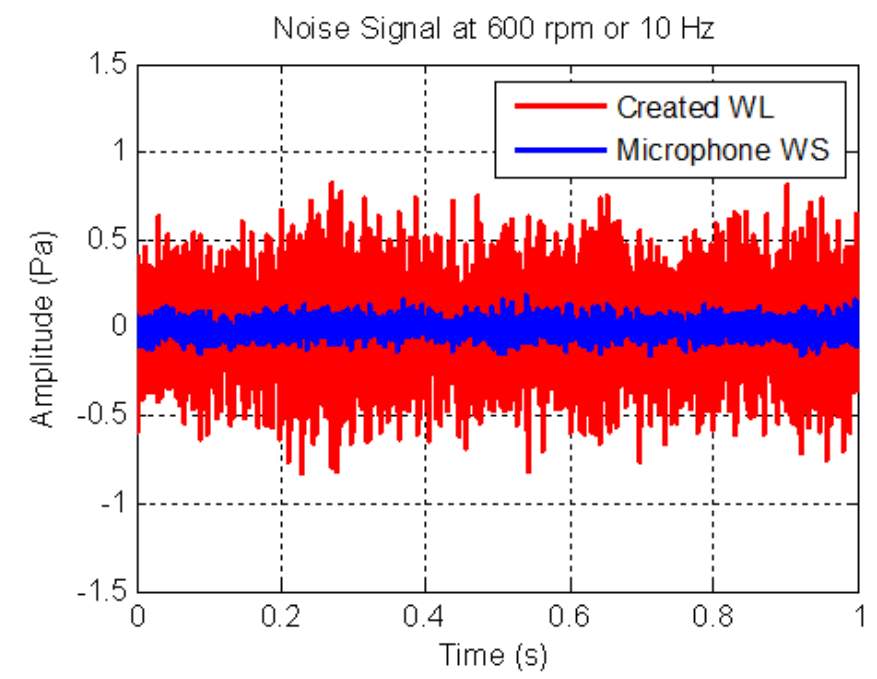

Figure 4. Noise signals on the created wireless and the standard wired microphone at 600/120 rpm

The testing result of noise signals or time domain between the created wireless and the standard wired microphone system shows that the signal from the created wireless is in accordance with or matches the standard noise signal as well as the signal from the standard wired microphone.

However, the amplitude of the noise signal from the created wireless is higher than that of the standard wired microphone noise signal. Therefore, the noise signal from the created wireless requires a calibration.

The calibrated noise signal on the created wireless at the speed of $600 / 120 \mathrm{rpm}$ is shown below.

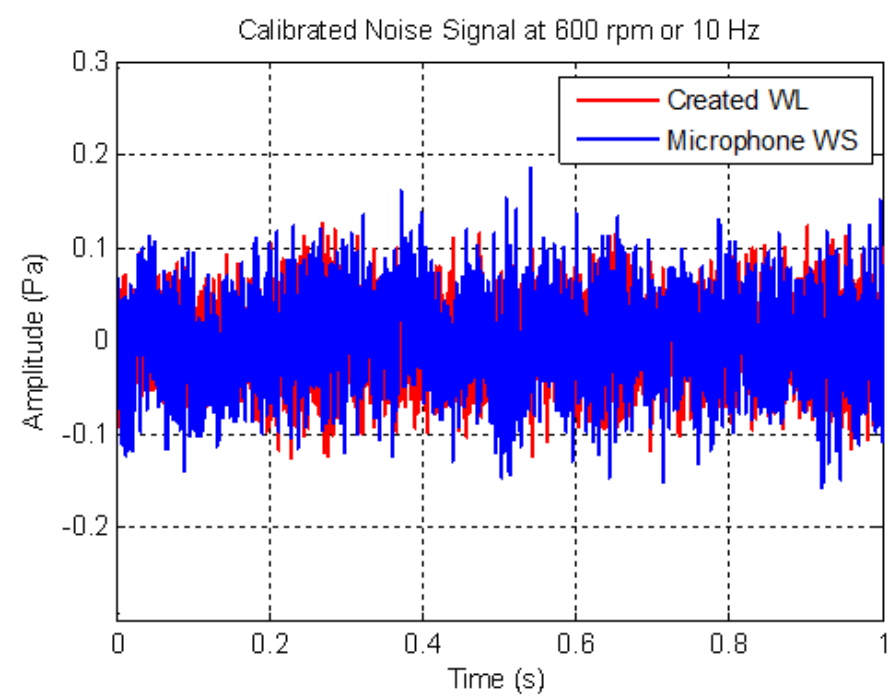

Figure 5. Calibrated noise signals on the created wireless and the wired microphone at $600 / 120 \mathrm{rpm}$ 
The calibrated noise signal from the created wireless at the speed of $1000 / 200 \mathrm{rpm}$ is shown in the following figure:

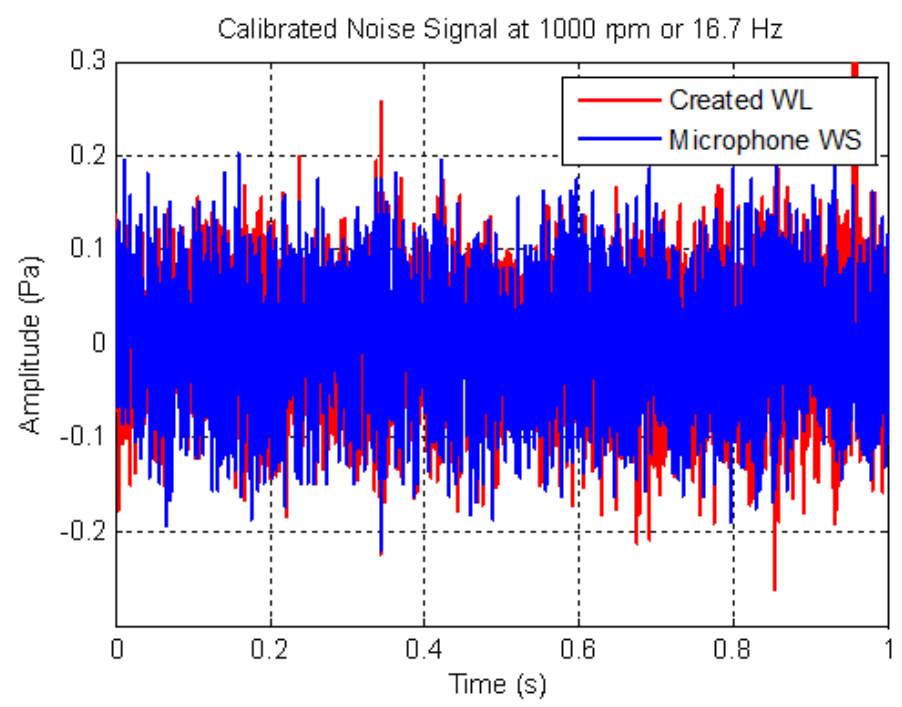

Figure 6. Calibrated noise signals on the created wireless and the standard wired microphone at the speed of $1000 / 200 \mathrm{rpm}$

The calibrated signal noise shows a similar shape and characteristics to each other. When the speed is increased, the maximum amplitude of the noise signal also increases.

\section{Noise signal result}

Noise spectrum on the gearbox transmission at the speed of $600 / 120 \mathrm{rpm}$ is shown in the following picture:

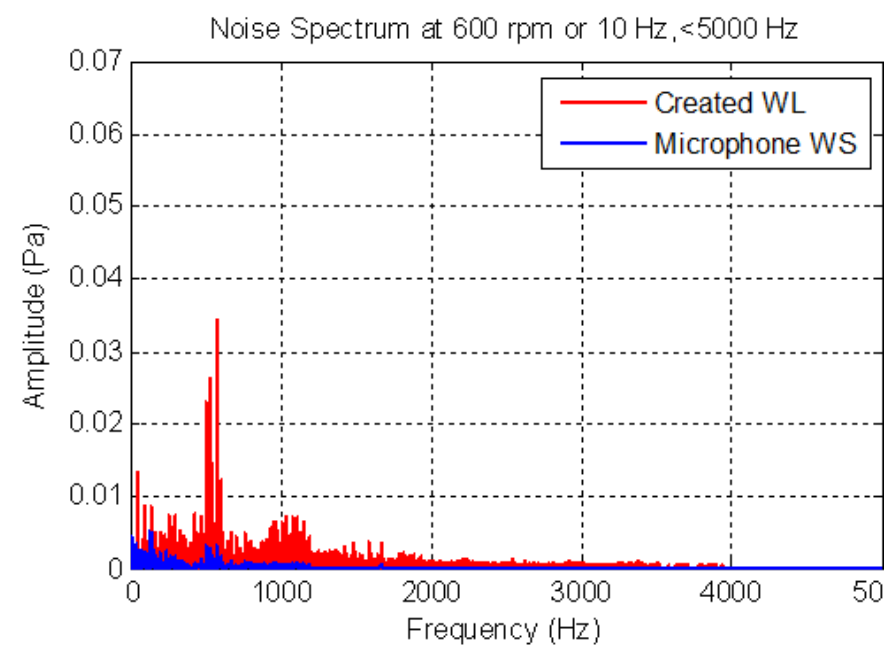

Figure 7. Noise spectrum on the created wireless and the wired microphone at the speed of 600/120 rpm in 1$5000 \mathrm{~Hz}$ 
Noise spectrum result on the gearbox transmission at the speed of $1000 / 200 \mathrm{rpm}$ is shown in the following figure:

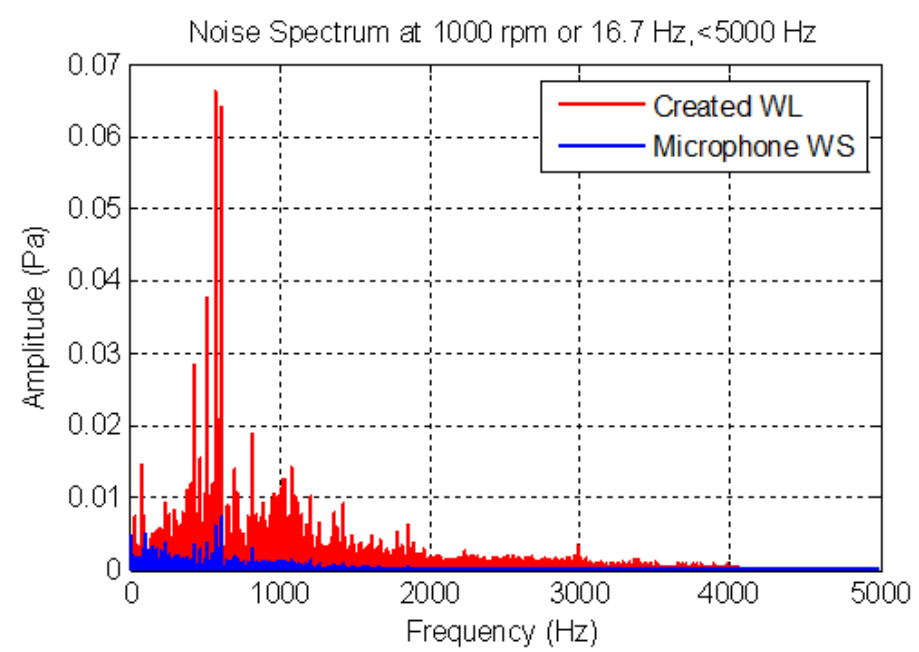

Figure 8. Noise spectrum on the created wireless and the wired microphone at the speed of 1000/200 rpm in 1$5000 \mathrm{~Hz}$

The noise spectrum or frequency domain on the created wireless and the standard wired microphone indicates that the noise spectrum on the created wireless matches that in general. The peak amplitude of noise spectrum on the created wireless is higher when compared to that on the standard wired microphone. Therefore, the noise spectrum on the created wireless is required for calibration. The highest amplitude occurs at about $500 \mathrm{~Hz}$.

The calibrated noise spectrum on the created wireless at the speed of $600 / 120 \mathrm{rpm}$ in $<5000$ $\mathrm{Hz}$ is shown below.

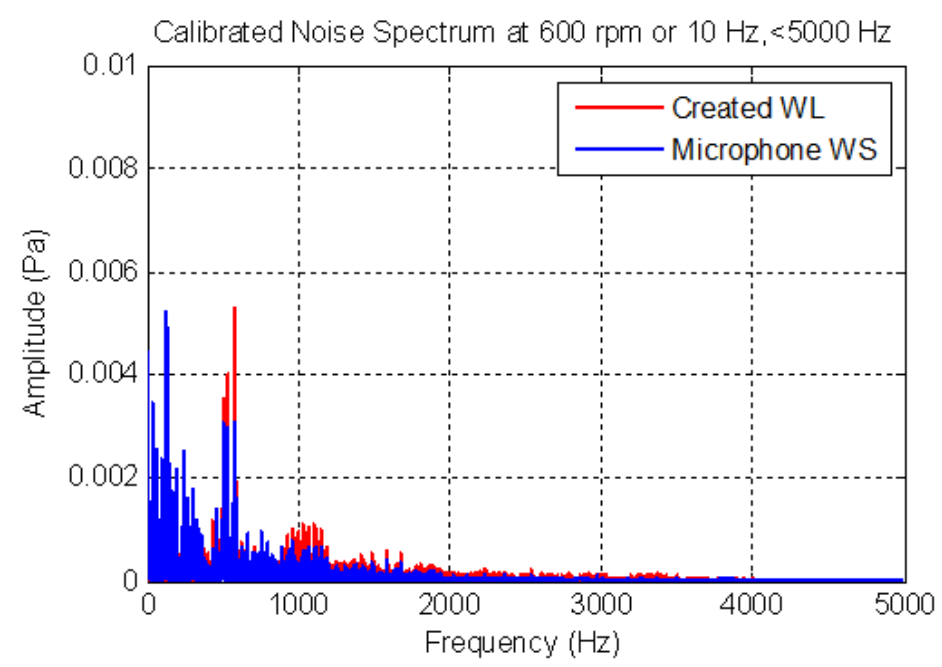

Figure 9. Calibrated noise spectrum on the created wireless and the wired microphone at the speed of $600 / 120 \mathrm{rpm}$ in $1-5000 \mathrm{~Hz}$ 
The calibrated noise spectrum on the created wireless at the speed of $1000 / 200 \mathrm{rpm}$ in $<5000 \mathrm{~Hz}$ is shown in the following figure:

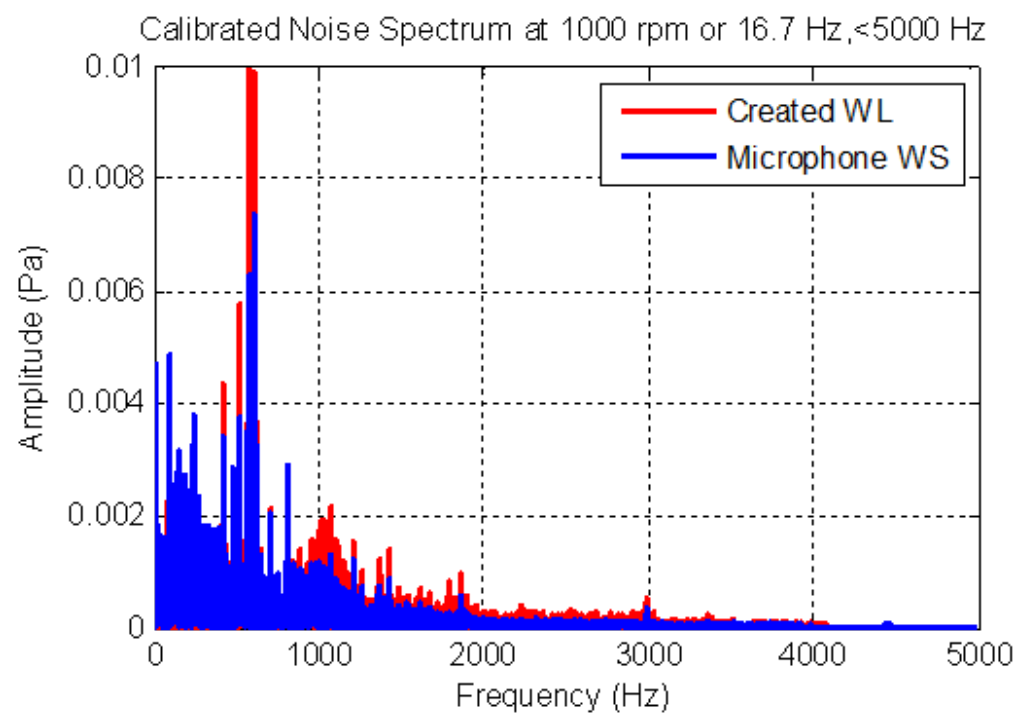

Figure 10. Calibrated noise spectrum on the created wireless and the wired microphone at the speed of $1000 / 200 \mathrm{rpm}$ in $1-5000 \mathrm{~Hz}$

The calibrated noise spectrum shows a similar pattern and characteristics at the frequency of $>750 \mathrm{~Hz}$. The amplitude and pattern noise spectrum at a low frequency indicate difference. The highest amplitude occurs at about $500 \mathrm{~Hz}$ or about 30 fundamental frequency.

Calibrated noise spectrum on the created wireless system at the speed of $600 / 120 \mathrm{rpm}$ at a low frequency $<375 \mathrm{~Hz}$ is shown in the following figure:

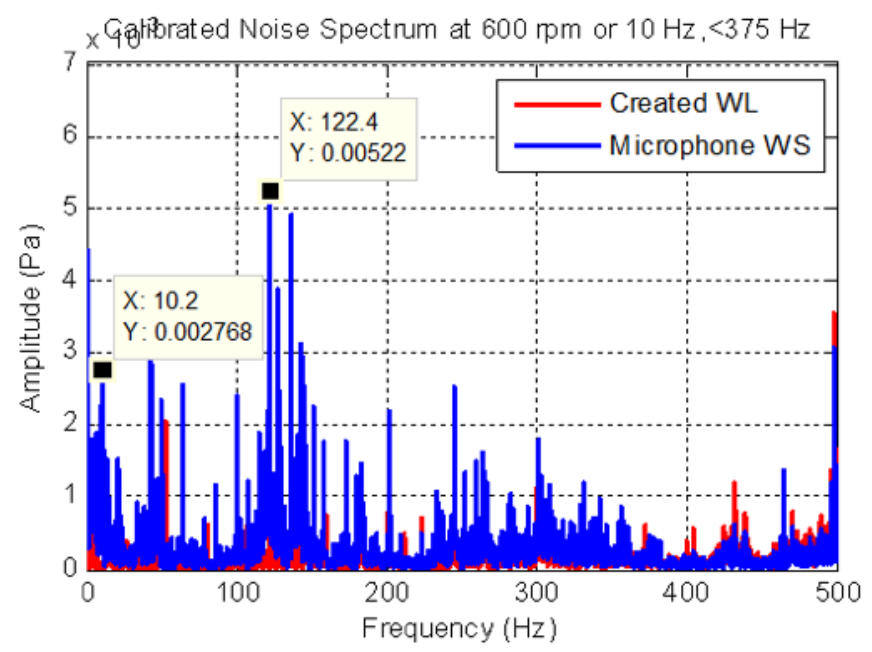

Figure 11. Calibrated spectrum noise on the created wireless and wire microphone at $600 / 120 \mathrm{rpm}$ in $1-375 \mathrm{~Hz}$ 
Calibrated noise spectrum on the created wireless at the speed of 1000/200 rpm in a low frequency $<375 \mathrm{~Hz}$ is shown in the following figure:

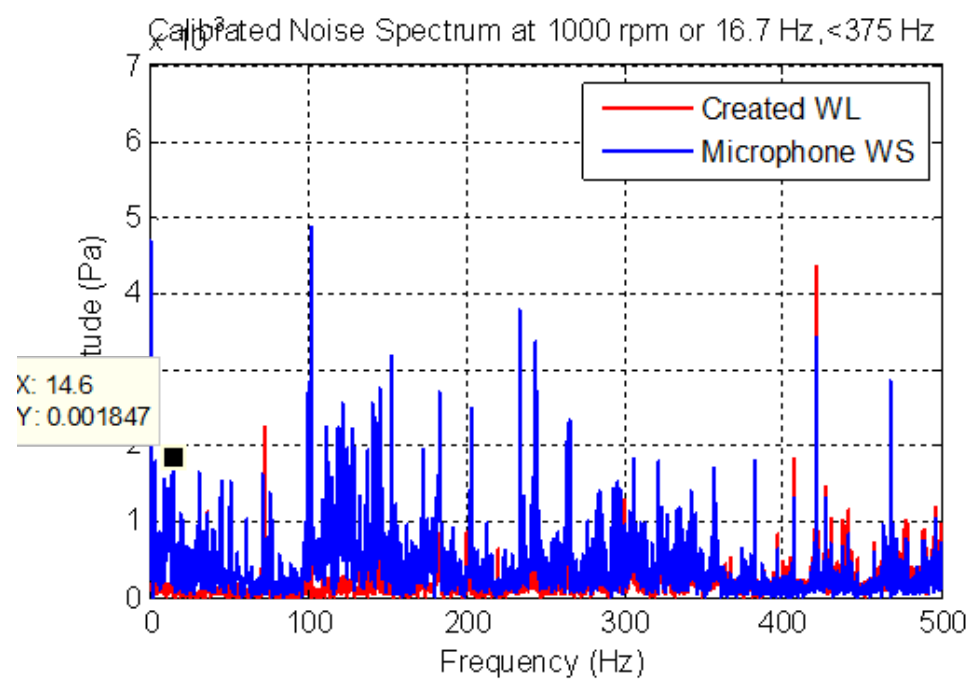

Figure 12. Calibrated noise spectrum on the created wireless and the wired microphone at the speed of $1000 / 200 \mathrm{rpm}$ in $1-375 \mathrm{~Hz}$

At a low frequency $<500 \mathrm{~Hz}$, noise spectrum on the created wireless and the standard wired microphone indicates that there is significant difference. Both of noise spectrums are not identical. The highest amplitude occurs at about 12 of fundamental frequency.

\section{CONCLUSIONS AND FUTURE INVESTIGATION}

This could be the basis for the design of penstock and selecting turbines that will be used. The conclusion of noise measurement tests on gearbox transmission using a created wireless and a standard wired system under a constant distance and constant load is as follows:

The noise signal using a created wireless system is in accordance with the expected noise signal. The amplitude of the noise signal and spectrum is higher than that on the standard wired system. Therefore, it requires calibration. After calibration, the noise signal and spectrum on the created wireless system matches that on the standard wired system, especially at a high frequency. When speed increases, the amplitude of the noise signal and noise spectrum also increases. The highest amplitude occurs at about 30 fundamental frequency. The highest amplitude at a low frequency occurs at about 12 fundamental frequency.

The investigation will be continued with a different distance between the transmitter and the receiver, and with a different load and different failure to get more information about noise characteristics using a created wireless system. 


\section{REFERENCES}

Arebi L, Fan Y, F Gu, Ball A. 2010. Investigation of Wireless Sensor on a Rotating Shaft and Its Potential for Machinery Condition Monitoring, $23^{\text {rd }}$ International Congress on Condition Monitoring an Diagnostic Engineering Management, June 28-July 2, Japan.

Barron F. 2003. Industrial Noise Control and Acoustic, Marcel Dekker Inc, New York.

Baydar N, Ball A. 2001. A Comparative Study of Acoustic and Vibration Signal in Detection of Gear Failures using Wigner-Ville Distribution, Mechanical System and Signal Processing, Academic Press, pp. 1091-1107.

Cook VG. 2012. Acoustic Monitoring of Machine Tool Health Using Transmitted Sound, Proceeding of the 2012 International Conference on Industrial Engineering and Operation Management, Istambul, Turkey, 3-6, pp. 1831-1839.

GST. 2010. General Purpose Piezoelectrics, YD-54251, Unit 1, 26 Fairholme Rd, Manchester, M20 4NT, UK.

Huskey A, Van Dam JM. 2010. Wind Turbine Generator System Acoustic Noise Test Report for The ARE 442 Wind Turbine, National Wind Technology Center, National Renewable Energy Laboratory, Colorado.

Kim M, Jang G, Lee C, Lim D (2010), Experimental Identification of Abnormal Noise and Vibration in a High Speed Polygon Mirror Scanner Motor due to Mechanical Contact of Plain Journal Bearing, Springer Verlag, Microsystem Technology 16, pp. 3-8.

Metwally SM, Khalil MI, Abouel Seoud SA. 2011. Noise Evaluation of Automotive AC Compressor, International Journal of Energy and Environtment, Volume 2, Issue 3, pp. 505-515.

Raharjo, P, Tesfa, B, Gu, F dan Ball, A, D, (2012), Comparative Study of the Monitoring of a Self Aligning Spherical Journal Bearing using Surface Vibration, Airborne Sound and Acoustic Emission, $25^{\text {th }}$ International Congress on Condition Monitoring and Diagnostic Engineering, IOP Publishing Journal of Physics Conference Series 364 (2012) 012035.

Ramroop, G., Liu, K, Gu, F, Payne S, Ball A., D. 2001. Airborne Acoustic Condition Monitoring of a Gearbox System, Electronic Proceeding of the $5^{\text {th }}$ Annual Maintenance and Reliability Conferrence, Gatlinburg, Tennessee, USA.

Rorrer, R. dan Juneja. V., 2002. Friction Induced Vibration and NoiseGeneration of Instrument Panel Material Pair, Elseiver, Tribology International, No. 35, pp. 523-531.

Wang, Y., Loh, K., J., Lynch, J. P., Frase, M., Law, K., Elgamal., A. 2006. Vibration Monitoring of the Voight Bridge using Wired and Wireless Monitoring System, The Proceeding of $4^{\text {th }}$ China-JapanUS Symposium on Structural Control and Monitoring, October 16-17. 